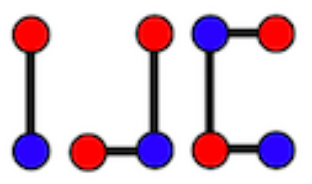

\title{
Dominating number of distance two of corona product of graphs
}

\author{
Reni Umilasari ${ }^{\mathrm{a}}$, Darmaji ${ }^{\mathrm{b}}$ \\ ${ }^{a}$ Department of Mathematics Education, Universitas Muhammadiyah Jember, Indonesia \\ ${ }^{b}$ Department of Mathematics, Institut Teknologi Sepuluh Nopember, Surabaya, Indonesia \\ reni.umilasari@gmail.com,darmaji@matematika.its.ac.id
}

\begin{abstract}
Dominating set $S$ in graph $G=(V, E)$ is a subset of $V(G)$ such that every vertex of $G$ which is not element of $S$ are connected and have distance one to $S$. Minimum cardinality among dominating sets in a graph $G$ is called dominating number of graph $G$ and denoted by $\gamma(G)$. While dominating set of distance two which denoted by $S_{2}$ is a subset of $V(G)$ such that every vertex of $G$ which is not element of $S$ are connected and have maximum distance two to $S_{2}$. Dominating number of distance two $\gamma_{2}(G)$ is minimum cardinality of dominating set of distance two $S_{2}$. The corona $G \odot H$ of two graphs $G$ and $H$ where $G$ has $p$ vertices and $q$ edges is defined as the graph $\mathrm{G}$ obtained by taking one copy of $G$ and $p$ copies of $H$, and then joining by an edge the $i-t h$ vertex of $G$ to every vertex in the $i-t h$ copy of $H$. In this paper, we determine the dominating number of distance two of paths and cycles. We also determine the dominating number of distance two of corona product of path and any graphs as well as cycle and any graphs.
\end{abstract}

Keywords: cycle, path, corona product, dominating number, dominating set

Mathematics Subject Classification : 05 C69

\section{Introduction}

Dominating set $S$ in a graph $G=(V, E)$ is a subset of $V(G)$ such that every vertex of $G$ which is not element of $S$ is connected and have distance one to $S$. The minimum cardinality among

Received: 15 July 2015， Revised: 26 July 2016, Accepted: 28 September 2016. 
dominating sets in a graph $G$ is called dominating number of a graph $G$ and denoted by $\gamma(G)$. Therefore, dominating number very closely related to dominating set.

The research on dominating set in a graph have been started in 1850 when the European chess players want to solve "dominating queens" problems. In this problem, dominating set is used to determine number of queens such that every square on a standard $8 \times 8$ chessboard is either occupied by a queen or can be directly attacked by a queen [9]. Research on dominating set and dominating number of a graph have been conducted such as c-dominating sets for families of graphs [9], dominating cartesian product of cycles [8], and total domination number of grid graph [4].

There are some applications of dominating set and dominating number of graph, i.e finding the school bus route and numbers of the bus stop such that students should not go too far from their home [7], finding number of fire engine that should be put in crossroads to cover all the region in case of fire and emergency first aid stations. Suppose that a natural disaster has struck some region consisting of many small villages. The vertices of a graph represent the villages in the region. An edge joining two vertices indicates that an emergency first aid station set up in one of the corresponding villages can also serve the other one. Then, a minimum dominating set of the graph would prescribe a way of serving the entire region with a minimum number of first aid stations [5]. But if the number of the emergency first aid stations is limited, it can be minimized by assuming one emergency first aid stations can dominate two nearest villages. This motivate us to define dominating number of distance two. Graphs that will be considered are path and cycle which are operated by corona product with any graphs. The corona $G \odot H$ of two graphs $G$ and $H$ where $G$ has $p$ vertices and $q$ edge is defined as the graph $\mathrm{G}$ obtained by taking one copy of $G$ and $p$ copies of $\mathrm{H}$, and then joining by an edge the $i-t h$ point of $G$ to every point in the $i-t h$ copy of $H[6]$.

Dominating set $S$ in a graph $G=(V, E)$ is a subset of $V(G)$ such that each vertex of $G$ which is not an element of $S$ is connected and have distance one to $S$. The minimum cardinality among dominating sets in a graph $G$ is called dominating number of a graph $G$ and denoted by $\gamma(G)$ [7]. While dominating set of distance two $S_{2}$ is a subset of $V(G)$ such that each vertex $G$ which is not an element of $S_{2}$ have distance less than or equals two to $S_{2}$. Dominating number of distance two, is denoted by $\gamma_{2}(G)$, is the minimum cardinality of dominating set of distance two. Figure 1 shows the minimum dominating sets of distance one and distance two, respectively. In Figure 1. (a) vertices $v_{2}$ and $v_{5}$ represent the vertices which are the element of the minimum dominating set of distance one, while vertex $u_{7}$ in figure 1 (b) represents the vertex which is the element of the minimum dominating set of distance two.

\section{Main Result}

We begin this section with the following theorem regarding to the dominating number of distance two of path and cycle.

Theorem 2.1. Path on $m$ vertices $P_{m}$ for any $m \geq 2$ has dominating number of distance two $\gamma_{2}\left(P_{m}\right)=\left\lceil\frac{m}{5}\right\rceil$ 


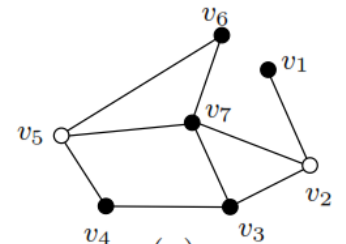

(a)

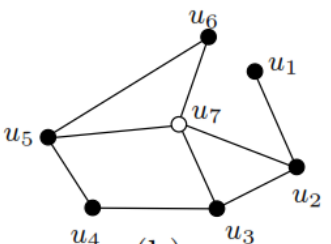

(b)

Figure 1. (a) Minimum dominating set of distance one (b) Minimum dominating set of distance two

Proof. Let $P_{m}$ be the Path with order $m$. Because the maximal degree of every vertex on $P_{m}$ is equal 2 , then one vertex can dominate at most 5 vertices with the distance less than or equal 2. Therefore the minimal number of vertices that can dominate by $m$ vertices are $\left\lceil\frac{m}{5}\right\rceil$. Thus, $\gamma_{2}\left(P_{m}\right) \geq\left\lceil\frac{m}{5}\right\rceil$.

We now show that $\left\lceil\frac{m}{5}\right\rceil$ is the minimal number element of $S_{2}$. Suppose $\gamma_{2}\left(P_{m}\right)=\left\lceil\frac{m}{5}\right\rceil-1$, then the maximal number of vertices that can be dominated on distance at most two are $5\left(\left\lceil\frac{m}{5}\right\rceil-1\right) \leq$ $5\left(\frac{m+4}{5}-1\right)=m-1$. Then, there are only $m-1$ vertices that can be dominated or at least one vertex of $V\left(P_{m}\right)$ cannot be dominated. It means that $\left|S_{2}\right|=\gamma_{2}\left(P_{m}\right) \neq\left\lceil\frac{m}{5}\right\rceil-1$. $\left\lceil\frac{m}{5}\right\rceil$.

Because $\left\lceil\frac{m}{5}\right\rceil$ is minimal number of vertices that can dominate all vertices on $P_{m}$, then $\gamma_{2}\left(P_{m}\right)=$

Theorem 2.2. Cycle on $n$ vertices $C_{n}$ for any $n \geq 3$ has dominating number of distance two $\gamma_{2}\left(C_{n}\right)=\left\lceil\frac{n}{5}\right\rceil$.

Proof. Cycle is regular 2 graph. Then for every $u_{i}$ element of $V\left(C_{n}\right), \operatorname{deg}\left(u_{i}\right)=2$. Because for every $u$ element $S_{2}, u$ can dominates at most 5 vertices with the distance less than or equal two and $\left|C_{n}\right|=n$, thus $\left|S_{2}\right| \geq\left\lceil\frac{n}{5}\right\rceil$.

To show that $\left\lceil\frac{n}{5}\right\rceil$ is the minimal number element of $S_{2}$, we suppose $\gamma_{2}\left(C_{n}\right)=\left\lceil\frac{n}{5}\right\rceil-1$. Then the maximal number of vertices that can be dominate on distance at most two are $5\left(\left\lceil\frac{n}{5}\right\rceil-1\right) \leq$ $5\left(\frac{n+4}{5}-1\right)=n-1$. It implies that only $n-1$ vertices that can be dominated by $S_{2}$ or at least one vertex of $C_{n}$ cannot be dominated. Therefore, $\left|s_{2}\right|=\gamma_{2}\left(C_{n}\right) \neq\left\lceil\frac{n}{5}\right\rceil-1$.

We can conclude that $\left\lceil\frac{n}{5}\right\rceil$ is minimal number of vertices that can dominate all vertices on $C_{n}$, then $\gamma_{2}\left(C_{n}\right)=\left\lceil\frac{n}{5}\right\rceil$.

For dominating number of distance two of corona product graphs will be begun with the following observations regarding to the relationship between a graph $G$ with the diameter at most 2 and its dominating number of distance two.

Observation 2.1. If a graph $G$ has diameter at most 2 , then $\gamma_{2}(G)=1$

Since the diameter of graph $G$ is at most 2, then the distance of any two vertices in $G$ is at most 2. By taking any $v_{i} \in V(G)$ as vertex element of dominating set of distance two $S_{2}(G)$, it implies that $d\left(v_{i}, V(G)\right) \leq 2$. Therefore, $\gamma_{2}(G)=1$. 
Some graphs which have diameter less than or equals two such as Complete $K_{n}$, Wheel $W_{n}$, Fan $F_{n}$, Friendship $W_{2}^{m}$, and Star $S_{n}$. Thus, $\gamma_{2}\left(K_{n}\right)=\gamma_{2}\left(W_{n}\right)=\gamma_{2}\left(F_{n}\right)=\gamma_{2}\left(W_{2}^{m}\right)=\gamma_{2}\left(S_{n}\right)=$ 1.

Observation 2.2. Let $G$ and $H$ be connected graphs of order $m$ and $n$, respectively. If diameter of $G$ is one $\gamma_{2}\left(G_{n} \odot H_{m}\right)=1$.

Because $G_{m}$ and $H_{n}$ are corona product graphs, then $\forall v_{i} \in G_{m}$ and $v_{i, j} \in H_{i}$ imply $d\left(v_{i}, v_{i, j}\right)=$ 1. Take arbitrary $v_{k} \in V\left(G_{m}\right)$ for $v_{k} \neq v_{i}$ as the vertex element of dominating set of distance two which implies $d\left(v_{k}, v_{i}\right)=1$, because the diameter of $G_{m}$ is equal 1 . While $d\left(v_{k}, v_{i, j}\right)=$ $d\left(v_{k}, v_{i}\right)+d\left(v_{i}, v_{i, j}\right)=2$ or any vertex in $G$ has maximal distance 2 to all vertices element $V\left(G_{m} \odot H_{n}\right)$, then $\gamma_{2}\left(G_{m} \odot H_{n}\right)=1$.

Graph with the diameter equals one for example Complete $K_{n}$. Then $\gamma_{2}\left(K_{n} \odot H\right)=1$, where $H$ is any graph.

We now present the dominating number of distance two of corona product of path with any graph and cycle with any graph as follows.

Theorem 2.3. Let $P_{m} \odot G_{n}$ be a corona product of path $P_{m}$ and any graphs $G_{n}$. Then dominating number of distance two $\gamma_{2}\left(P_{m} \odot G_{n}\right)=\left\lceil\frac{m}{3}\right\rceil$ for $m \geq 2$.

Proof. Suppose $V\left(P_{m} \odot G_{n}\right)=\left\{v_{1}, v_{2}, \ldots, v_{m}\right\} \cup\left\{v_{i, j} \mid 1 \leq i \leq m, 1 \leq j \leq n\right\}$, then $\left|P_{m} \odot G_{n}\right|=m n+m$. The cases below show three posibilities of minimum number of vertices which are the element of dominating number of distance two on $P_{m} \odot G_{n}$.

Case 1: $S_{2} \in V\left(G_{i}\right)$

For every $v_{i, j}$ element of $V\left(G_{i}\right), v_{i, j}$ can dominate at most $n+3$ vertices. Then the maximal number of vertices which are the element of dominating number of distance two is $\frac{m n+m}{n+3}$, thus $\left|S_{2}\right| \leq \frac{m n+m}{n+3}$.

Case 2: $S_{2} \in V\left(P_{m}\right)$

For every $v_{i}$ element of $V\left(P_{m}\right), v_{i}$ can dominate at most $3 n+5$ vertices. Then the maximal number of vertices which are the element of dominating number of distance two is $\frac{m n+m}{3 n+5}$, thus $\left|S_{2}\right| \leq \frac{m n+m}{3 n+5}$.

Case 3: $S_{2} \in V\left(G_{i}\right) \cup V\left(P_{m}\right)$

For every $v_{i}$ element of $V\left(P_{m}\right)$ and $v_{i, j}$ element of $V\left(G_{i}\right)$, then two vertices can dominate at most $(n+3)+(3 n+5)$ vertices. Thus $\left|S_{2}\right| \leq \frac{2(m n+m)}{(n+3)+(3 n+5)}=\frac{m n+m}{n+4}$.

By three cases above $\frac{m n+m}{3 n+5} \leq \frac{m n+m}{n+4} \leq \frac{m n+m}{n+3}$, then we can take $V\left(P_{m}\right)$ as the vertex element of $S_{2}$. Because $\frac{m n+m}{3 n+5}=\frac{m(n+1)}{3(n+1)+2}<\frac{m(n+1)}{3(n+1)}=\frac{m}{3}$, then the interval or distance of every vertex element of $S_{2}$ is equal 3. Because $\left|S_{2}\right|$ must be integer number, then minimal number of vertices which be the element of dominating number of distance two of $V\left(P_{m} \odot G_{n}\right)$ are $\left\lceil\frac{m}{3}\right\rceil$. Therefore $\gamma_{2}\left(P_{m} \odot G_{n}\right) \geq\left\lceil\frac{m}{3}\right\rceil$ 
Next, we show that $\left\lceil\frac{m}{3}\right\rceil$ is the minimal number element of $S_{2}$. Let $\left|S_{2}\right|=\left\lceil\frac{m}{3}\right\rceil-1$, then the maximal number of vertices which are the element of $S_{2}$ is

$$
\begin{aligned}
\left(\left\lceil\frac{m}{3}\right\rceil-1\right)(3 n+3) & \leq\left(\frac{m+2}{3}-1\right)(3 n+3) \\
& =m n+m-n-1 \\
& <m n+m .
\end{aligned}
$$

It can conclude that not all vertices can be dominated. Therefore, $\left|S_{2}\right| \neq\left\lceil\frac{m}{3}\right\rceil-1$ and $\left\lceil\frac{m}{3}\right\rceil$ is minimum dominating number of $P_{m} \odot G_{n}$. Thus it is proven that $\gamma_{2}\left(P_{m} \odot G_{n}\right)=\left\lceil\frac{m}{3}\right\rceil$.

Theorem 2.4. Let $C_{n} \odot H_{m}$ be a corona product of cycle $C_{n}$ and any graphs $H_{m}$. Then dominating number of distance two $\gamma_{2}\left(C_{n} \odot H_{m}\right)=\left\lceil\frac{n}{3}\right\rceil$ for $n \geq 3$.

Proof. Let $V\left(C_{n} \odot H_{m}\right)=\left\{u_{1}, u_{2}, \ldots, u_{n}\right\} \cup\left\{u_{i, j} \mid 1 \leq i \leq n, 1 \leq j \leq m\right\}$, then $\left|C_{n} \odot H_{m}\right|=$ $n m+n$. Some cases below show three posibilities of vertices which element of minimum dominating set of graph $C_{n} \odot H_{m}$.

Case 1: $S_{2} \in V\left(H_{i}\right)$

For every $u_{i, j}$ element of $V\left(H_{i}\right)$, vertex $u_{i, j}$ can dominate at most $m+3$ vertices. Then the maximal number of vertices which are the element of dominating number of distance two is $\frac{n m+n}{m+3}$, then $\left|S_{2}\right| \leq \frac{n m+n}{m+3}$.

Case 2: $S_{2} \in V\left(C_{n}\right)$

For every $u_{i}$ elemen $V\left(C_{n}\right)$, vertex $u_{i}$ can dominate at most $3 m+5$ vertices. Then the maximal number of vertices which are the element of dominating number of distance two is $\frac{n m+n}{3 m+5}$, then $\left|S_{2}\right| \leq \frac{n m+n}{3 m+5}$.

Case 3: $S_{2} \in V\left(H_{i}\right) \cup V\left(C_{n}\right)$

For every $u_{i}$ element of $V\left(C_{n}\right)$ and $u_{i, j}$ element of $V\left(H_{i}\right)$, then two vertices can dominate at most $(m+3)+(3 m+5)$ vertices, thus it implies $\left|S_{2}\right| \leq \frac{2(n m+n)}{(m+3)+(3 m+5)}=\frac{n m+n}{m+4}$.

From case 1 up to $3, \frac{n m+n}{3 m+5} \leq \frac{n m+n}{m+4} \leq \frac{n m+n}{m+3}$. Then we can take the minimal number of $S_{2}$ are vertex set of Cycle $C_{n}$. Because $\frac{n m+n}{3 m+5}=\frac{n(m+1)}{3(m+1)+2}<\frac{n(m+1)}{3(m+1)}=\frac{n}{3}$, then the interval or distance of every vertex element of $S_{2}$ is equal 3. We know that $\left|S_{2}\right|$ must be integer number and distance of every vertex element of $S_{2}$ is equal 3 , then minimal number of vertices which are the element of dominating number of distance two of $V\left(C_{n} \odot H_{m}\right)$ is $\left\lceil\frac{n}{3}\right\rceil$, thus $\gamma_{2}\left(C_{n} \odot H_{m}\right) \leq\left\lceil\frac{n}{3}\right\rceil$.

Therefore, to show that $\left\lceil\frac{n}{3}\right\rceil$ is minimal dominating number, we can take $\left|S_{2}\right|=\left\lceil\frac{n}{3}\right\rceil-1$. And it makes the maximal number of vertices which are the element of $S_{2}$ is

$$
\begin{aligned}
\left(\left\lceil\frac{n}{3}\right\rceil-1\right)(3 m+3) & \leq\left(\frac{n+2}{3}-1\right)(3 m+3) \\
& =n m+n-m-1 \\
& <n m+n .
\end{aligned}
$$


We can conclude that not all vertices can be dominated. Therefore, $\left|S_{2}\right| \neq\left\lceil\frac{n}{3}\right\rceil-1$ and $\left\lceil\frac{n}{3}\right\rceil$ is minimum dominating number of $C_{n} \odot H_{m}$. Then $\gamma_{2}\left(C_{n} \odot H_{m}\right)=\left\lceil\frac{n}{3}\right\rceil$.

\section{Conclusion}

We conclude this paper with an open problem regarding to the dominating number of corona product of any two graphs $G$ and $H$, that is

Open problem 4.1. For any graph $\mathrm{G}$ on $m$ vertices and any graph $H$ on $n$ vertices, find $\gamma_{2}(G \odot H)$.

\section{References}

[1] G. Chartrand, L. Lesniak, Graphs and Digraph, $3^{\text {rd }}$ edition, Chapman \& Hall/CRC, (1996),26 Boundaru Row, London SE1 8HN, UK.

[2] C. Go, S. Canoy, Domination in The Corona and Join of Graphs, International Mathematical Forum 6 (16) (2011), 763-771.

[3] W. Goddard, M. A. Henning, Independent Domination in Graphs: A Survey and Recent Results, (2006), University of Johannesburg, South Africa.

[4] S. Gravier, Total Domination Number of Grid Graph, Discrete Applied Mathematics, 121 (2002), 119-128.

[5] J. Gross, J. Yellen, Graph Theory and Its Applications, Chapman \& Hall/CRC, FL 334872742, (2006), Boca Raton, London.

[6] F. Harary, R. Frucht, On The Corona Of Two Graphs, Aequationes Mathematicae, (1970), 322-325.

[7] W. Haynes, Teresa, Fundamental of Dominations in Graphs, (1996), New York: Marcel Dekker, Inc.

[8] S. Klavžar, Dominating Cartesian Product of Cycles, Discrete Applied Mathematics, 59 (1995), 129-136.

[9] K. Snyder, c-'Dominating Sets for Families of Graphs, (2011), University of Mary Washington. 